\title{
EFECTO DE LA ORIENTACIÓN DE LA LADERA SOBRE LA ESTRUCTURA POBLACIONAL Y ECOMORFOLOGÍA dE NEOBUXBAUMIA TETETZO (CACTACEAE)
}

\author{
Víctor López-Gómez' , Paulina Zedillo-Avelleyra, SoonYoo Anaya-Hong, Enrique \\ GonZÁlez-Lozada y ZenÓn CANO-Santana
}

Laboratorio de Interacciones y Procesos Ecológicos, Facultad de Ciencias, Universidad Nacional Autónoma de México, México, D.F.

${ }^{1}$ Autor para la correspondencia: abies226@yahoo.com.mx

\begin{abstract}
Resumen: La orientación de la ladera modifica las condiciones microclimáticas de los sitios. A pesar de este conocido patrón son pocas las evidencias de su efecto sobre las poblaciones de plantas y son aún más escasas para las cactáceas. En este estudio se determinó el efecto de la orientación de la ladera norte y sur sobre algunos atributos poblacionales (el área total del tallo, la densidad y la estructura de tamaños) y la ecomorfología (número y orientación de las ramas) de Neobuxbaumia tetetzo (Cactaceae) mayores a $1.5 \mathrm{~m}$ de alto en el Valle de Zapotitlán, Puebla. En la ladera sur se presentó una mayor densidad de tetechos y una mayor tendencia a orientar sus ramas hacia el noreste, que los tetechos de la ladera norte. Mientras que en la ladera norte los tetechos mostraron más ramificaciones y hubo un menor número de tetechos de tamaño pequeño en comparación con la población de tetechos de la ladera sur. Se concluye que la orientación de la ladera afectó claramente la densidad, la estructura de tamaños y la ecomorfología de $N$. tetetzo; sin embargo, se requieren de estudios sobre los parámetros microclimáticos del sitio para poder hacer una generalización. Palabras clave: densidad, estructura de tamaños, orientación de las ramas, tetechos.
\end{abstract}

\begin{abstract}
Slope orientation modifies field microclimatic conditions. Despite this well-known pattern, there is few empirical evidence of its influence on plant populations, and even lesser for cactus populations. Our goals were to determine the effect of $\mathrm{N}$ and $\mathrm{S}$ slope orientation on some population attributes (total stem area, density and size structure) and ecomorphology (number branch and orientation) of Neobuxbaumia tetetzo (Cactaceae) taller than $1.5 \mathrm{~m}$ in Valle de Zapotitlán, Puebla. In southerly slope, $N$. tetetzo showed higher density and a higher tendency of branches to northeast orientation than N. tetetzo in northerly slope. In the northern slope cacti had a higher number of branches than in the southern one. We conclude that slope orientation showed a remarkable effect on density, size structure, and ecomorphology of N. tetetzo; nevertheless, it is needed to obtain microclimatic records at the study site to clarify our understanding about slope orientation effect on N. tetezo population.
\end{abstract}

Key words: branch orientation, density, giant columnar cactus, size structure.

$\mathbf{L}$ a disponibilidad de agua en las zonas áridas está determinada, entre otros factores, por el efecto de la orientación de las laderas. En el hemisferio norte, las laderas con exposición sur reciben mayor radiación solar (casi seis veces más) que sus contrapartes con orientación norte; siendo las laderas con exposición norte más húmedas en comparación con aquellas con orientación sur (Nobel, 1982; GonzálezHidalgo et al., 1996; del Castillo, 2000; Valverde-Padilla, 2002; Auslander, 2003; Mazzola et al., 2008). En sitios con déficit hídrico, la orientación de ladera tiene un gran efecto en las condiciones ambientales, por tanto este factor es determinante en el desarrollo y la estructura de las comunidades vegetales (Carter y Klinka, 1990; del Castillo, 2000).

Los organismos que habitan en laderas con orientaciones contrastantes, pueden presentar diferencias morfológicas y fisiológicas; las cuales, les permiten sobrevivir a las condiciones microclimáticas que se presentan en cada ladera (Nevo, 2001). El efecto de la orientación de la ladera en las plantas se ha registrado en su morfología (Auslander, 2003) y en su estructura vegetal (González-Hidalgo et al., 1996; 
del Castillo, 2000; Mazzola et al., 2008). Por ejemplo, las hojas del arbusto Pistacia lentiscus (Anacardiaceae) fueron más grandes en laderas norte que en laderas sur (Auslander, 2003). En el matorral xerófilo del Valle de Zapotitlán (Puebla), se encontró que los arbustos dominaron las laderas norte. Mientras que en las laderas sur fueron más abundantes las formas rosetófilas y presentaron una mayor diversidad de formas de vida (Valverde-Padilla, 2002).

La familia Cactaceae es un componente ecológico importante en las zonas áridas y semiáridas de América, ya que presenta una alta riqueza de especies y las plantas de esta familia son el refugio y el alimento de muchas especies (Alanís-Flores y Velazco-Macías, 2008). Estas plantas presentan adaptaciones para tolerar el déficit hídrico (Hernández-González, 2007); por ejemplo, los saguaros pueden producir una mayor cantidad de ramificaciones en las laderas con una orientación que maximiza la efectividad de la insolación (Drezner, 2003).

A pesar de que existen varios estudios que muestran los efectos de la orientación de la ladera sobre las comunidades vegetales (Whittaker y Marks, 1975; Ortiz-Pulido y RicoGray, 2006), existen pocas evidencias de su efecto sobre la estructura poblacional y morfología de las plantas. Asimismo, el conocimiento de los factores que determinan los patrones de crecimiento de las cactáceas columnares aún es limitado. Este hecho, es particularmente relevante, si se considera que el Valle de Tehuacán-Cuicatlán es el sitio con el mayor número de especies de cactáceas columnares en México (DávilaAranda et al., 1993; Valiente-Banuet et al., 1996). El objetivo de este trabajo fue conocer el efecto de la orientación de la ladera sobre algunos aspectos poblacionales (estructura de tamaños, densidad y área basal total) y la morfología (número y orientación de ramas) de la cactácea columnar Neobuxbaumia tetetzo en Zapotitlán Salinas, Puebla.

\section{Materiales y métodos}

Sitio de estudio. El estudio se realizó en el Jardín Botánico "Helia Bravo-Hollis" (18 19' 50.3” N, 97²7'32.1” O) dentro de la Reserva de la Biosfera Tehuacán-Cuicatlán, en el municipio de Zapotitlán Salinas, Puebla. El sitio tiene una altitud de 1,503 m (Rodríguez, 1990). El promedio anual de precipitación en la región varía de los 250 a los $500 \mathrm{~mm}$, presentándose la mayor precipitación entre junio y septiembre. La vegetación es un matorral xerófilo en donde dominan las plantas de la familia Cactaceae (Arias-Toledo et al., 2001). Sistema de estudio. Neobuxbaumia tetetzo (Coulter) Backeberg, es una cactácea columnar conocida como tetecho que alcanza alturas superiores a los $10 \mathrm{~m}$, produce de dos a cinco ramas por individuo adulto a partir del tallo principal. Los tallos y las ramas se caracterizan por ser cilíndricos, presentar costillas y carecer de hojas; sus espinas tienen de 5 a $20 \mathrm{~mm}$ de largo y se dirigen hacia abajo. Esta planta crece en laderas de cerros formando parte de la vegetación de bosques espinosos y bosques tropicales caducifolios (Bravo-Mendoza et al., 2007).

Muestreo. En marzo de 2010 se trazaron 15 cuadros de 10 $\times 10 \mathrm{~m}$ de manera sistemática en una ladera orientada al norte y en una ladera orientada al sur, en un mismo cerro, dentro del Jardín Botánico "Helia Bravo-Hollis". En cada cuadro se contó el número de tetechos (densidad), el número y la orientación de las ramas a partir del tallo principal de cada planta columnar, y también se midió el perímetro del tallo principal a $40 \mathrm{~cm}$ del nivel del suelo. Sólo se registraron los individuos con una altura superior a $1.5 \mathrm{~m}$, con la intención de reducir la variación que podrían aportar los individuos más pequeños. Ya que se ha registrado que las características de los tetechos menores de $1 \mathrm{~m}$ de altura están fuertemente influenciados por las condiciones microclimáticas que proporciona su planta nodriza (Bravo-Mendoza et al., 2007). Con el perímetro del tallo ( $p b)$ de cada tetecho se calculó el área basal total $(A t)$ para cada cuadro, considerando que la base de estas cactáceas tiene una forma circular. Para realizar la estructura de tamaños se utilizó el perímetro del tallo de los tetechos y se determinó el número de categorías de tamaño con la regla de Silk (1979) con la siguiente fórmula:

$$
n_{c}=5 \log (N)
$$

donde $n_{c}$ es el número de categorías de tamaño y $N$ es el número de registros.

Análisis de datos. Para comparar las medias de la densidad, el área total de todos los tallos por cuadro y el número de ramas entre las laderas norte y sur, se realizaron pruebas de $t$ para muestras independientes. La densidad y el número de ramas por individuo fueron transformados con la fórmula:

$$
x^{\prime}=\sqrt{x+0.05}
$$

donde $x$ ' es el registro transformado y $x$ es el registro sin transformar; ya que éstas son variables discretas que no se distribuyen normalmente (Zar, 2010).

Para determinar la diferencia de las medias angulares de la orientación de las ramas de los tetechos entre laderas, se aplicó una prueba de comparación de dos medias angulares de Watson y Williams (Zar, 2010). Como los datos presentaron distribuciones de frecuencias bimodales, primero se calculó las medias angulares para datos axiales con la finalidad de obtener una distribución de frecuencias unimodal y después se realizó una prueba de uniformidad circular de Rayleigh para cada caso como lo sugiere Zar (2010).

Para determinar si la estructura de tamaños de los tetechos difiere entre las laderas con orientación norte y sur, se realizó una prueba de $\chi^{2}$ utilizando 13 categorías de tamaños del área del tallo de las plantas $(A t)$. Además se realizaron análisis de residuos estandarizados para determinar si las frecuencias observadas eran significativamente mayores o menores que las frecuencias esperadas (Siegel, 1986). Las pruebas estadísticas de $t$ y de $\chi^{2}$ fueron realizadas con el programa Statistica versión 8.0 (StatSoft, 2007). 


\section{Resultados}

El tamaño de muestra fue de 493 tetechos (183 en la ladera norte y 310 en la sur). La ladera sur presentó una densidad de tetechos mayor que la ladera norte $(t=4.36$, g.l. $=28, P=$ $<0.001$ ) (Cuadro 1). El número de ramas de los tetechos fue significativamente mayor en la ladera norte que en la ladera sur $(t=2.01$, g.l. $=493, P=0.04)$. Las orientaciones de las ramas de los tetechos no presentaron una distribución circular uniforme en ambas laderas (ladera norte, $z=20.38$, g.l. = 450, $P<0.001$; ladera sur, $z=46.79$, g.l. $=622, P<0.001$ ). La medias angulares fueron significativamente diferentes entre laderas ( $t=2.28$, g.l. $=1070, P=0.02$; Cuadro 1). No se encontraron diferencias significativas en las medias del área total de los tallos de los tetechos entre las laderas $(t=$ 0.94 , g.l. $=28, P=0.35$; Cuadro 1).

La estructura de tamaños de los tetechos fue diferente entre las laderas $\left(\chi^{2}=24.56\right.$, g.l. $\left.=12, P=0.01\right)$. En la ladera norte se presentó la mayor frecuencia de individuos en la cuarta categoría de tamaño $\left(459.1-592.0 \mathrm{~cm}^{2}\right)$, mientras que en la ladera sur se observó en la segunda categoría $\left(193.1-326.0 \mathrm{~cm}^{2}\right)$. La frecuencia de individuos observados en la segunda categoría de tamaños $\left(193.1-326.0 \mathrm{~cm}^{2}\right)$ en la ladera norte fue significativamente menor que los valores esperados (Figura 1).

\section{Discusión}

La orientación de la ladera afectó la densidad y la ecomorfología de los tetechos mayores a $1.5 \mathrm{~m}$ de alto en Zapotitlán
Salinas, Puebla. La ladera con orientación sur presentó una población de tetechos más densa y en la ladera norte se registró un menor número de plantas pequeñas de lo que se esperaría por azar; estas evidencias sugieren que la ladera sur presenta las condiciones más favorables para los tetechos; ya que se ha registrado que las laderas con orientación sur presentan una mayor radiación solar en comparación con las laderas con orientación norte (Auslander, 2003; Mazzola et al., 2008). Asimismo, nuestros registros sobre los tetechos que presentaron una mayor cantidad de ramas en la ladera norte que la ladera sur, sugieren que los tetechos de la ladera norte reciben una cantidad de luz por abajo del óptimo (Cuadro 1), ya que se ha registrado que el aumento en la

Cuadro 1. Comparación de algunos atributos de la población (densidad y área total de los tallos por cuadro) y la morfología (orientación y número de las ramas) de Neobuxbaumia tetetzo ( \pm error estándar) en dos laderas con orientaciones contrastantes (norte y sur) de un mismo cerro del Jardín Botánico "Helia Bravo-Hollis", Zapotitlán de las Salinas, Puebla. El parámetro de la dispersión de los datos para la medida angular de la orientación de las ramas es la desviación estándar circular. Letras diferentes denotan diferencias significativas, $P<0.05$.

\begin{tabular}{lrr}
\hline \multicolumn{1}{c}{ Variable } & \multicolumn{2}{c}{ Orientación } \\
& Norte & \multicolumn{1}{c}{ Sur } \\
\hline Densidad (ind./100 $\left.\mathrm{m}^{2}\right)$ & $12.20 \pm 1.43^{\mathrm{b}}$ & $20.67 \pm 1.45^{\mathrm{a}}$ \\
Número de ramas & $2.47 \pm 0.28^{\mathrm{a}}$ & $2.00 \pm 0.24^{\mathrm{b}}$ \\
Área basal total $\left(\mathrm{cm}^{2} / 100 \mathrm{~m}^{2}\right)$ & $213.98 \pm 15.50^{\mathrm{a}}$ & $187.59 \pm 15.50^{\mathrm{a}}$ \\
Media angular de la & $80 \pm 50.39^{\mathrm{a}}$ & $72.5 \pm 46.07^{\mathrm{b}}$ \\
orientación de las ramas $\left(^{\circ}\right)$ & & \\
\hline
\end{tabular}

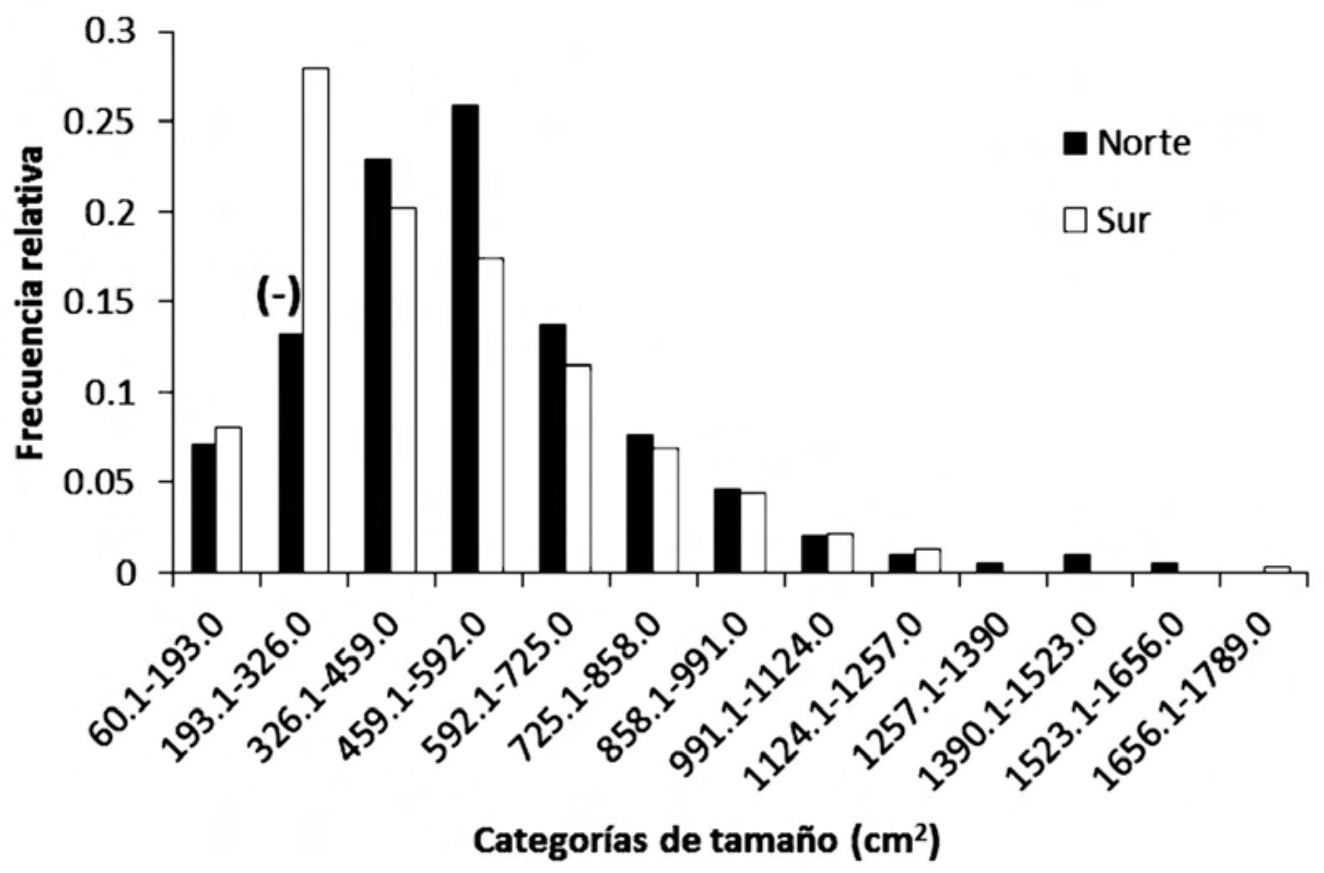

Figura 1. Estructuras de tamaños de Neobuxbaumia tetetzo en laderas con orientación norte y sur. El signo negativo entre paréntesis señala que las frecuencias observadas fueron significativamente menores a las esperadas para esa categoría de tamaño $(P<0.05)$. 

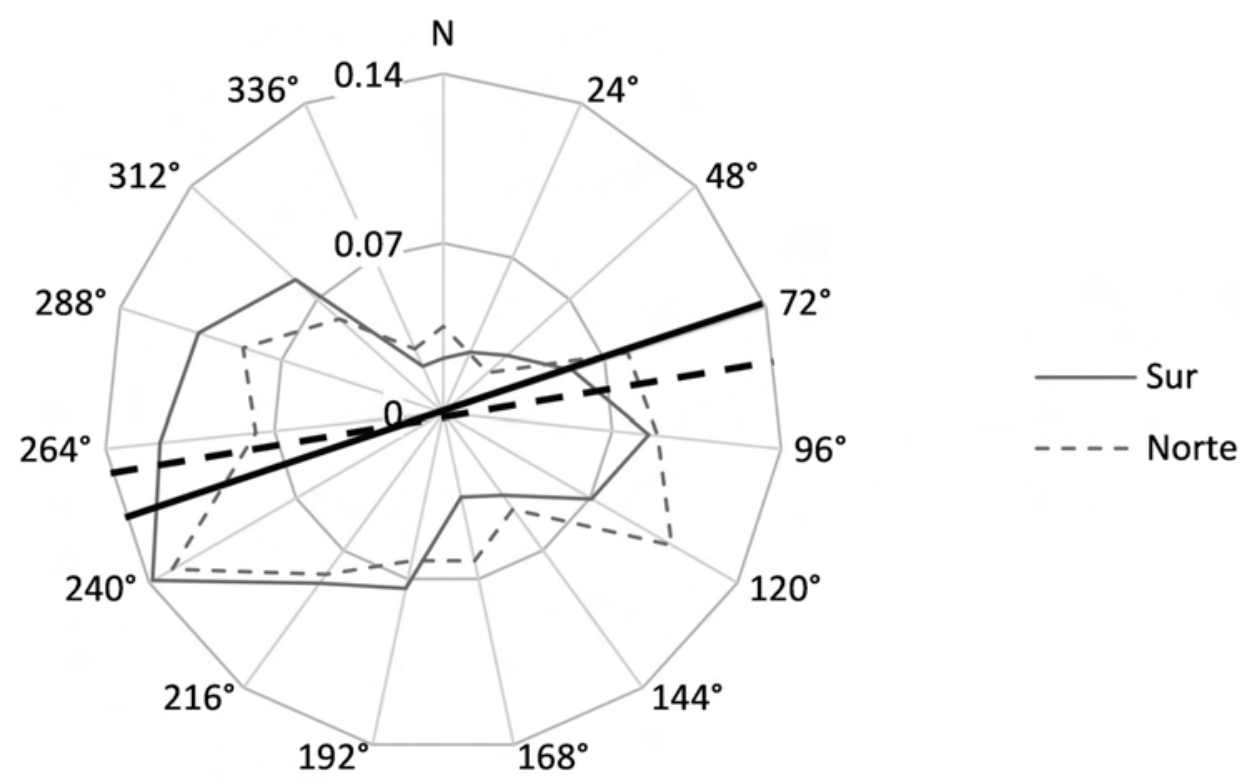

Figura 2. Medias angulares y distribución de radial de las frecuencias relativas de las ramas de Neobuxbaumia tetetzo en dos laderas con orientaciones contrastantes (sur y norte), en el Jardín Botánico "Helia Bravo-Hollis", Zapotitlán de las Salinas, Puebla. La prueba de Watson y Williams señaló que las medias angulares fueron significativamente diferentes.

cantidad de ramas en cactáceas columnares es una estrategia para compensar una baja radiación solar (Drezner, 2003).

La alta densidad de tetechos que se registró en la ladera sur, coincide con el trabajo de Mata-González et al. (2002) realizado en un desierto de Nuevo México, quienes encontraron una mayor densidad (i.e. más del doble) de varias especies de plantas suculentas en la ladera con orientación sur que en la ladera norte.

La diferencia que encontramos en las orientaciones de las ramas de los tetechos entre las laderas norte y sur, nos señala que este contraste es muy sutil; por tanto, para corroborar que este patrón no sea un artefacto, sugerimos que esta tendencia se debe de probar en otros cerros. Por otro lado, se corroboró la tendencia de las ramas de los tetechos a orientarse hacia el este y el oeste en ambas laderas (Figura 2), tal como se ha registrado en la cactácea columnar Carnegiea gigantea (Drezner, 2003). Este patrón se ha adjudicado a la tendencia de las plantas a orientar sus estructuras fotosintéticas hacia el este y oeste como una estrategia para aprovechar la mayor radiación solar cuando la temperatura es más favorable a lo largo del día (Nobel, 1981; Barker y Adams, 1997). Para nuestro sistema de estudio se podría especular que las mejores condiciones de temperatura para las ramas con orientación hacia el este se presentan en el amanecer, mientras que para las ramas con orientación al oeste sería en el ocaso.

Los resultados obtenidos no permiten hacer una generalización del efecto de la ladera sobre los cambios en la densidad y en la morfología de los tetechos en esta zona de estudio. Para probar nuestras hipótesis sugerimos evaluar las diferencias microambientales entre laderas, enfocándose principalmente en la radiación solar, la temperatura y la humedad. Asimismo se debe determinar la influencia de otros factores que podrían estar afectando a la estructura poblacional y morfología de los tetechos, como son las características del suelo (Martínez-Berdeja y Valverde, 2008) y las interacciones con otros organismos (Valiente-Banuet y Ezcurra, 1991; García-Chávez et al., 2010).

Se concluye que la orientación de la ladera afectó la densidad, el número y la orientación de las ramas de los individuos de $N$. tetetzo mayores a $1.5 \mathrm{~m}$ de alto en Zapotitlán Salinas, Puebla.

\section{Agradecimientos}

Los autores agradecen a Iván Castellanos-Vargas y a Ixchel Sarahí González-Ramírez por su revisión y comentarios que ayudaron a mejorar el manuscrito, así como a Daniela Fernández y Fernández y al grupo 5222 de Ecología I (2010-II) de la Facultad de Ciencias de la UNAM, por su apoyo en la colecta de datos.

\section{Literatura citada}

Alanis-Flores G.J. y Velasco-Macías C.G. 2008. Importancia de las cactáceas como recurso natural en el Noreste de México. Ciencia UANL 11:5-11.

Arias-Toledo A.A., Valverde-Valdés M.T. y Reyes-Santiago J. 2000. Las Plantas de la Región de Zapotitlán Salinas, Puebla. Instituto Nacional de Ecología/Red de desarrollo Sostenible 
A.C./ Universidad Nacional Autónoma de México, México, D.F.

Auslander M., Nevo E. e Inbar M. 2003. The effects of slope orientation on plant growth, developmental instability and susceptibility to herbivores. Journal of Arid Environments 55:405-416.

Barker D.H. y Adams W.W. 1997. The xanthophyll cycle and energy dissipation in differently oriented faces of the cactus Opuntia macrorhiza. Oecologia 109: 353-361.

Bravo-Mendoza M., Espinosa-Cantú A., Castellanos-Vargas I. y Cano-Santana Z. 2007. Tamaño de Neobuxbaumia tetetzo y longitud de sus espinas apicales en un gradiente de luz bajo Mimosa luisana, un arbusto nodriza. Acta Botanica Mexicana 79:69-80.

Carter R.E. y Klinka K.1990. Relationships between growingseason soil water-deficit, mineralizable soil nitrogen and site index of coastal Douglas fir. Forest Ecology and Management 30:301-311.

Dávila-Aranda P., Villaseñor-Ríos J.L., Medina-Lemos R., Ramírez-Roa A., Salinas-Tovar A., Sánchez-Ken J. y Tenorio-Lezama P. 1993. Listados Florísticos de México X. Flora del Valle de Tehuacán Cuicatlán. Instituto de Biología. Universidad Nacional Autónoma de México. México, D.F.

del Castillo R.F. 2000. Composición y estructura de una nopalera bajo situaciones contrastantes de exposición de ladera y herbivoría. Boletín de la Sociedad Botánica de México 65:5-22.

Drezner T.D. 2003. Branch direction in Carnegiea gigantea (Cactaceae): Regional patterns and the effect of nurse plants. Journal of Vegetation Science 14:907-910.

García-Chávez J., Sosa V.J. y Montaña C. 2010. Variation in postdispersal predation of cactus seeds under nurse plant canopies in three plant associations of a semiarid scrubland in central Mexico. Journal of Arid Environments 74:54-62.

González-Hidalgo J.C., Bonet A.y Echeverría M.T. 1996. Efecto de la orientación de la ladera sobre algunas comunidades arbustivas del semiárido central de la depresión del Ebro. Mediterranea 15:21-31.

Hernández-González O. 2007. Estudio comparativo en la ecofisiología de cáctaceas columnares (Tribu: Pachycereeae). Tesis doctoral, Instituto de Ecología, A.C. Xalapa, Veracruz. 68 pp.

Martínez-Berdeja A. y Valverde M.T. 2008. Growth response of three globose cacti to radiation and soil moisture: An experimental test of the mechanism behind the nurse effect. Journal of Arid Environments 72:1766-1774.

Mata-González R., Pieper R.D. y Cárdenas M.M. 2002. Vegetation patterns as affected by aspect and elevation in small desert mountains. The Southwestern Naturalist 47:440-448.

Mazzola M.B., Kin A.G., Morici E.F., Babinec F.J.y Tamborini G. 2008. Efecto del gradiente altitudinal sobre la vegetación de las sierras Lihue Calel (La Pama, Argentina). Boletín de la Sociedad Argentina de Botánica 43:103-119.

Nevo E. 2001. Evolution of genome-phenome diversity under environmental stress. Proceedings of the National Academy of Sciences of the United States of America 98:6233-6240.

Nobel P.S. 1981. Influences of photosynthetically active radiation on cladode orientation, stem tilting, and height of cacti. Ecology 62:982-990.

Nobel P.S. 1982. Orientation, PAR interception, and nocturnal acidity increases for terminal cladodes of a widely cultivated cactus, Opuntia ficus-indica. American Journal of Botany 69:14621469.

Ortiz-Pulido R. y Rico-Gray V. 2006. Seed dispersal of Bursera fagaroides (Burseraceae): the effect of linking environmental factors. The Southwestern Naturalist 51:11-21.

Rodríguez-Acosta M. 1990. Tehuacán, reloj del tiempo vegetal. Elementos, Ciencia y Cultura 14:26-36.

Rzedowski J. 1978. Vegetación de México. Limusa, México, D.F.

Siegel S. 1986. Estadística no Paramétrica: Aplicada a las Ciencias de la Conducta. Trillas, México, D.F.

Silk, J. 1979. Statistical Concepts in Geography. Harper Collins Publishers Ltd. Londres.

Statsoft. 2007. Statistica v. 8: data analysis software system. Statsoft Inc., Tulsa.

Valiente-Banuet A. y Ezcurra E. 1991. Shade as a cause of the association between the cactus Neobuxbaumia tetetzo and the nurse plant Mimosa luisana in the Tehuacan valley, Mexico. Journal of ecology 79:961-971.

Valiente-Banuet A., Arizmendi M.C., Rojas-Martínez A. y Domínguez-Canseco L. 1996. Ecological relationships between columnar cacti and nectar-feeding bats in Mexico. Journal of Tropical Ecology 12:103-119.

Valverde-Padilla P.L. 2002. Cambios en la abundancia, dominancia y diversidad de formas de vida vegetales entre laderas norte y sur en el Valle semiárido de Zapotitlán, Puebla. ContactoS 45:45-50.

Whittaker R.H. y Marks P.L. 1975. Methods of assessing terrestrial productivity. En: Lieth H. y Whittaker R.H. Eds. Primary Productivity of the Biosphere, pp.55-118, Springer-Verlag, Nueva York.

Zar J.H. 2010. Biostatistical Analysis. Prentice Hall, Nueva Jersey.

Recibido: 20 de octubre de 2011

Aceptado: 28 de marzo de 2012 